\title{
BI-LIPSCHITZ EMBEDDING OF PROJECTIVE METRICS
}

\author{
LEONID V. KOVALEV
}

\begin{abstract}
We give a sufficient condition for a projective metric on a subset of a Euclidean space to admit a bi-Lipschitz embedding into Euclidean space of the same dimension.
\end{abstract}

\section{INTRODUCTION}

A metric $d$ on a convex domain $\Omega \subseteq \mathbb{R}^{n}$ is called projective (sometimes Desarguesian) provided that the equality $d(x, z)+d(z, y)=d(x, y)$ holds if and only if $z$ is a convex combination of $x$ and $y$. Equivalently, a metric is projective if line segments are unique geodesics. Two well-known classes of such metrics are strictly convex normed spaces and Hilbert geometries on convex sets [8]. A different, integralgeometric construction of projective metrics was introduced by Busemann [6]. Let $\mathcal{H}$ be the set of all hyperplanes, i.e., $(n-1)$-dimensional affine subspaces, in $\mathbb{R}^{n}$. For a set $E \subseteq \mathbb{R}^{n}$ denote by $\pi E \subseteq \mathcal{H}$ the set of all hyperplanes that intersect $E$. Throughout the paper $n \geq 2$.

Definition 1.1. Let $\Omega \subseteq \mathbb{R}^{n}, n \geq 2$, be a convex domain. Suppose $\nu$ is a positive Borel measure on $\mathcal{H}$ such that

- $\nu(\pi E)=0$ when $E$ is a one-point subset of $\Omega$;

- $\nu(\pi E)>0$ when $E$ is a line segment in $\Omega$;

- $\nu(\pi E)<\infty$ for every compact set $E$ contained in $\Omega$.

Then

$$
d_{\nu}(x, y)=\nu(\pi[x, y])
$$

is a Busemann-type projective metric on $\Omega$.

The fact that $d_{\nu}$ is a projective metric is immediate from the definition. In the converse direction, Pogorelov [15], Ambartzumian [3] and Alexander [1] showed that every projective metric on $\mathbb{R}^{2}$ arises from Busemann's construction. See [2, 7, 14] for an historical overview and other results towards Hilbert's fourth problem, which asks for a characterization of projective metrics.

The fact that the Euclidean metric on $\mathbb{R}^{n}$ can be constructed as in Definition 1.1 is a consequence of the classical Crofton formula (e.g., [17]). We write $d_{e}$ for the Euclidean metric. The main result of this paper is the following sufficient condition for $\left(\Omega, d_{\nu}\right)$ to admit a bi-Lipschitz embedding into $\mathbb{R}^{n}$.

Theorem 1.2. In the notation of Definition 1.1, suppose that the identity map $\mathrm{id}:\left(\Omega, d_{\nu}\right) \rightarrow\left(\Omega, d_{e}\right)$ is locally $\eta$-quasisymmetric. Then $\left(\Omega, d_{\nu}\right)$ is bi-Lipschitz

Received by the editors December 30, 2013 and, in revised form, March 15, 2014.

2010 Mathematics Subject Classification. Primary 30L05; Secondary 30C65, 51M10.

Key words and phrases. bi-Lipschitz embedding, projective metric, quasisymmetric map.

This work was supported by the NSF grant DMS-0968756. 
equivalent to $\left(\Omega^{\prime}, d_{e}\right)$ for some domain $\Omega^{\prime} \subseteq \mathbb{R}^{n}$. Furthermore, if $\Omega=\mathbb{R}^{n}$, then $\Omega^{\prime}=\mathbb{R}^{n}$.

The assumption of Theorem 1.2 is that there exists a modulus of quasisymmetry $\eta$ (see Definition 2.3) such that every point of $\left(\Omega, d_{\nu}\right)$ has a neighborhood in which the identity map is $\eta$-quasisymmetric. This is a weaker assumption than id being quasisymmetric in $\Omega$. Section 3 presents a more precise version of Theorem 1.2 , namely Theorem 3.7

Theorem 1.2 highlights the difference between the Busemann construction (placing a weight on the space of hyperplanes) and the conformal deformation (placing a weight on the Euclidean space itself). For the latter, the analog of Theorem 1.2 fails, as was demonstrated by Semmes [18] in dimensions $n \geq 3$ and by Laakso [10. in dimension $n=2$. In particular, Laakso constructed a nonsmooth conformal deformation of $\mathbb{R}^{2}$ such that the resulting space is not bi-Lipschitz embeddable into any uniformly convex Banach space, despite the identity map to $\left(\mathbb{R}^{2}, d_{e}\right)$ being quasisymmetric.

After the definitions and preliminary results are collected in Section 2 , the proof of Theorem 1.2 is given in 3 . It employs a construction of quasiconformal maps that simultaneously extends two previously known approaches [5, 13]; this connection is discussed in $\$ 4$ The concluding Section 5 presents some open problems.

\section{Preliminaries}

Let $\Omega$ and $\nu$ be as in Definition 1.1. Fix a point $o \in \Omega$. For a hyperplane $H \in \mathcal{H}$ that does not pass through $o$, let $n(H)$ be the unit normal vector to $H$ that points out of the halfspace containing $o$. Define

$$
f_{\nu}(x)=\int_{\pi[o, x]} n(H) d \nu(H), \quad x \in \Omega .
$$

The choice of basepoint $o$ is immaterial: it contributes only an additive constant to $f_{\nu}$ (see the proof of Lemma 2.1 below). Note that $f_{\nu}(o)=0$.

Given a nonzero vector $v$ and a hyperplane $H \in \mathcal{H}$, let $\alpha(v, H) \in[0, \pi / 2]$ be the smaller angle between $H$ and the line determined by $v$. For example, $\alpha(v, H)=\pi / 2$ when $v$ is orthogonal to $H$. This notation will be used often in the sequel.

Lemma 2.1. For all $x, y \in \Omega$,

$$
\left|f_{\nu}(x)-f_{\nu}(y)\right| \leq \nu(\pi[x, y])
$$

and

$$
\left\langle f_{\nu}(x)-f_{\nu}(y), x-y\right\rangle=|x-y| \int_{\pi[x, y]} \sin \alpha(x-y, H) d \nu(H) .
$$

Proof. For $x, y \in \Omega$ we have

$$
f_{\nu}(x)-f_{\nu}(y)=\int_{\pi[0, x] \backslash \pi[0, y]} n(H) d \nu(H)-\int_{\pi[0, y] \backslash \pi[0, x]} n(H) d \nu(H) .
$$

Since the symmetric difference of $\pi[0, x]$ and $\pi[0, y]$ agrees with $\pi[x, y]$ up to a $\nu$-null set, (2.2) follows.

When $H \in \pi[0, x] \backslash \pi[0, y]$, the inner product $\langle n(H), x-y\rangle$ is positive. When $H \in \pi[0, y] \backslash \pi[0, x]$, this inner product is negative. Therefore, taking the inner 
product of both sides in (2.4) with $x-y$ yields

$$
\begin{aligned}
\left\langle f_{\nu}(x)-f_{\nu}(y), x-y\right\rangle & =\int_{\pi[x, y]}|\langle n(H), x-y\rangle| d \nu(H) \\
& =|x-y| \int_{\pi[x, y]} \sin \alpha(x-y, H) d \nu(H)
\end{aligned}
$$

proving (2.3). In particular,

$$
\left|f_{\nu}(x)-f_{\nu}(y)\right| \geq \int_{\pi[x, y]} \sin \alpha(x-y, H) d \nu(H) .
$$

According to Lemma 2.1, $f_{\nu}$ is an injective 1-Lipschitz map from $\left(\Omega, d_{\nu}\right)$ to $\mathbb{R}^{n}$. In general, it is not bi-Lipschitz. However, it satisfies a weaker noncollapsing property.

Lemma 2.2. There is a constant $c=c(n)>0$ such that for every cube $Q \subset \Omega$,

$$
\operatorname{diam} f_{\nu}(Q) \geq c \operatorname{diam}_{\nu} Q
$$

where $\operatorname{diam}_{\nu}$ is the diameter with respect to the metric $d_{\nu}$.

Proof. Let $H$ be a hyperplane that intersects $Q$ and does not meet any of its vertices. Write $a$ for the edgelength of $Q$. Since $Q$ contains a ball of diameter $a$, the projection of $Q$ onto the line $H^{\perp}$ has diameter at least $a$. This diameter is realized by projections of two vertices that are separated by $H$; call them $x$ and $y$. Since $|x-y| \leq a \sqrt{n}$, it follows that

$$
\alpha(x-y, H) \geq \sin ^{-1}(1 / \sqrt{n}) .
$$

For every pair $x, y$ of distinct vertices of $Q$, let $S_{x y}$ be the set of hyperplanes $H$ that separate $x$ from $y$ and satisfy (2.7). By the above, the union of $S_{x, y}$ over all such pairs $\{x, y\}$ is $\pi Q$. Counting the number of pairs of vertices, we conclude that there exists a pair $\{x, y\}$ such that $\nu\left(S_{x, y}\right) \geq 4^{-n} \nu(\pi Q)$. For such a pair, (2.5) yields

$$
\left|f_{\nu}(x)-f_{\nu}(y)\right| \geq 4^{-n} n^{-1 / 2} \nu(\pi Q) \geq 4^{-n} n^{-1 / 2} \operatorname{diam}_{\nu} Q
$$

Definition 2.3. Let $\eta:[0, \infty) \rightarrow[0, \infty)$ be a homeomorphism, called a modulus of quasisymmetry below. A topological embedding $f: X \rightarrow \mathbb{R}^{n}$ of a metric space $X$ into $\mathbb{R}^{n}$ is called $\eta$-quasisymmetric if for every triple of distinct points $a, b, x \in X$,

$$
|f(x)-f(a)| \leq \eta(t)|f(x)-f(b)| \quad \text { where } \quad t=\frac{d_{X}(x, a)}{d_{X}(x, b)} .
$$

When there is no need to emphasize the modulus of quasisymmetry $\eta$, we simply say that $f$ is quasisymmetric. Bi-Lipschitz maps are quasisymmetric but not conversely. The foundational facts about quasisymmetric maps in metric spaces are presented in [9].

In what follows we use standard notation $B(a, r)=\left\{x \in \mathbb{R}^{n}:|x-a|<r\right\}$ and $\bar{B}(a, r)=\overline{B(a, r)}$. Unspecified multiplicative constants $C$ and $c$ are always positive, and may differ from one line to another. 


\section{UNIFORM TRANSVERSALITY}

Informally, a measure $\nu$ on $\mathcal{H}$ is uniformly transverse if it not tightly concentrated on hyperplanes that are nearly parallel to some line. The precise statement follows.

Definition 3.1. Let $\nu$ and $\Omega$ be as in Definition 1.1. We say that $\nu$ is uniformly transverse on $\Omega$ if there exists $\kappa>0$ such that

$$
\int_{\pi[x, y]} \sin \alpha(x-y, H) d \nu(H) \geq \kappa \nu(\pi[x, y])
$$

for all $x, y \in \Omega$.

Some remarks are in order. When a line segment $[x, y] \subset \Omega$ is divided into subsegments, both sides of (3.1) are additive with respect to such partition. Thus, it suffices to verify (3.1) for sufficiently short segments. Also, (3.1) is equivalent to the existence of $\tau>0$ such that

$$
\nu(\{H \in \pi[x, y]: \alpha(x-y, H) \geq \tau\}) \geq \tau \nu(\pi[x, y]) .
$$

Indeed, (3.2) obviously implies (3.1) with $\kappa=\tau \sin \tau$. Conversely, if (3.1) holds, then letting $\tau=\kappa / 2$ we find that

$$
\begin{aligned}
\kappa \nu(\pi[x, y]) & \leq \int_{\pi[x, y]} \sin \alpha(x-y, H) d \nu(H) \\
& \leq \tau \nu(\pi[x, y])+\nu(\{H \in \pi[x, y]: \sin \alpha(x-y, H) \geq \tau\}),
\end{aligned}
$$

hence (3.2) holds.

When $\Omega=\mathbb{R}^{n}$ in Definition 3.1 we simply say that $\nu$ is uniformly transverse. The following result relates uniform transversality to the quasisymmetry of the identity map.

Proposition 3.2. If the map id: $\left(\Omega, d_{\nu}\right) \rightarrow\left(\Omega, d_{e}\right)$ is locally $\eta$-quasisymmetric, then $\nu$ is uniformly transverse.

Proof. As observed above, it suffices to consider a short segment $[x, y]$. Let $r=$ $|x-y|$. The assumption of quasisymmetry implies that by taking sufficiently small $c=c(\eta, n)>0$, we can ensure that any cube $Q$ with center $x$ and edgelength $c r$ satisfies

$$
\operatorname{diam}_{\nu} Q \leq \frac{1}{2 \cdot 4^{n}} d_{\nu}(x, y) .
$$

Since $\nu$-almost every hyperplane crossing $Q$ separates a pair of its vertices (and there are $2^{n}$ vertices), it follows that there is a pair of vertices $u, v$ such that $d_{\nu}(u, v) \geq 4^{-n} \nu(\pi Q)$. Thus, (3.3) implies

$$
\nu(\pi Q) \leq \frac{1}{2} \nu(\pi[x, y]) .
$$

For any hyperplane $H \in \pi[x, y] \backslash \pi Q$ the angle $\alpha(x-y, H)$ is bounded from below by a constant that depends only on $c$. Since the set of such hyperplanes has $\nu$-measure at least $\frac{1}{2} \nu(\pi[x, y])$, the claim follows.

For any $\nu$ as in Definition 1.1. the map $f_{\nu}$ is monotone in the sense that $\left\langle f_{\nu}(x)-\right.$ $\left.f_{\nu}(y), x-y\right\rangle \geq 0$ whenever $x \neq y$; this is a consequence of (2.3). In fact, it satisfies a stronger property defined below. 
Definition 3.3. Let $\Omega$ be a convex domain in $\mathbb{R}^{n}$. A map $f: \Omega \rightarrow \mathbb{R}^{n}$ is called cyclically monotone if

$$
\sum_{k=1}^{m}\left\langle f\left(x_{k}\right), x_{k+1}-x_{k}\right\rangle \leq 0
$$

holds for all $m \geq 2$ and all $x_{1}, \ldots, x_{m} \in \Omega$. Here $x_{m+1}=x_{1}$.

Observe that for $m=2$ the inequality (3.5) amounts to monotonicity. The concept of cyclic monotonicity is motivated by the fact that cyclically monotone maps are precisely subsets of subgradients of convex functions [16, Theorem 24.8]. In particular, every continuous cyclically monotone map is the gradient of a $C^{1}$ convex function.

Proposition 3.4. The map $f_{\nu}$ in (2.1) is cyclically monotone.

Proof. Since the inequality (3.5) is additive with respect to $f$, it suffices to verify it for the integrand in (2.1). Fix a hyperplane $H$ not passing through 0 . It can be described by the equation $H=\{x:\langle x, n(H)\rangle=c\}$ for some $c>0$. Let $g(x)=$ $n(H)$ if $H$ separates $x$ from 0 , and $g(x)=0$ otherwise. The function $U(x)=$ $\max (c,\langle x, n(H)\rangle)$ is convex and its subgradient $\partial U$ satisfies $g(x) \in \partial U(x)$ for every $x \in \mathbb{R}^{n}$. Therefore, $g$ is cyclically monotone, and so is $f_{\nu}$.

Yet another concept of monotonicity comes into play when $\nu$ is uniformly transverse.

Definition 3.5. Let $\Omega$ be a convex domain in $\mathbb{R}^{n}$. For a fixed $\delta>0$, a map $f: \Omega \rightarrow \mathbb{R}^{n}$ is called $\delta$-monotone if

$$
\langle f(x)-f(y), x-y\rangle \geq \delta|f(x)-f(y)||x-y|
$$

holds for all $x, y \in \Omega$.

Neither cyclic monotonicity nor $\delta$-monotonicity imply each other.

Proposition 3.6. The following are equivalent:

(i) $\nu$ is uniformly transverse;

(ii) $f_{\nu}$ is $\delta$-monotone;

(iii) $f_{\nu}$ is a locally $\eta$-quasisymmetric embedding of $\left(\Omega, d_{e}\right)$ into $\mathbb{R}^{n}$.

The equivalence is quantitative in the sense that the constants involved in each statement depend only on one another and on the dimension $n$.

Proof. The equivalence of (i) and (ii) is established by the identify (2.3). By [12, Theorem 6 ], every $\delta$-monotone map is locally quasisymmetric; more precisely, there exists a modulus of quasisymmetry $\eta$ that depends only on $\delta$, such that $f$ is $\eta$ quasisymmetric in every ball $B(x, r)$ such that $B(x, 2 r) \subset \Omega$. This shows (ii) $\Longrightarrow$ (iii). For the converse, observe that $f_{\nu}$ is continuous and cyclically monotone; therefore, it can be written as the gradient of a differentiable convex function $u: \Omega \rightarrow \mathbb{R}$. By [12, Lemma 18], if the gradient of a convex function is locally $\eta$-quasisymmetric, it is $\delta$-monotone where $\delta$ depends only on $\eta$. This completes the proof.

By virtue of Proposition 3.2, Theorem 1.2 is a consequence of the following more precise statement. 
Theorem 3.7. If $\nu$ is uniformly transverse on $\Omega$, then the map $f_{\nu}$ defined by (2.1) is a bi-Lipschitz embedding of $\left(\Omega, d_{\nu}\right)$ into $\mathbb{R}^{n}$. Furthermore, if $\Omega=\mathbb{R}^{n}$, then $f_{\nu}(\Omega)=\mathbb{R}^{n}$.

Proof of Theorem 3.7. From (2.2) we see that $f_{\nu}$ is Lipschitz. The reverse inequality $\left|f_{\nu}(x)-f_{\nu}(y)\right| \geq c d_{\nu}(x, y)$ follows by combining (2.5) and (3.1).

By Proposition $3.6 f_{\nu}$ is a locally $\eta$-quasisymmetric embedding of $\left(\Omega, d_{e}\right)$ into $\mathbb{R}^{n}$, therefore it is a quasiconformal map (e.g., 9]). It is well-known that $\mathbb{R}^{n}$ cannot be quasiconformally mapped to its proper subdomain [20, Theorem 17.4]. Thus, in the case $\Omega=\mathbb{R}^{n}$ we have $f_{\nu}(\Omega)=\mathbb{R}^{n}$.

\section{EXAMPLES}

A convenient way to introduce measures on the space of hyperplanes $\mathcal{H}$ is to push them forward from a space where it is easier to construct measures. For example, there is a natural surjection $\mathbb{R}^{n} \times S^{n-1} \rightarrow \mathcal{H}$ given by $\Phi(a, v)=\{x:\langle x, v\rangle=\langle a, v\rangle\}$. Let $\omega$ be the normalized volume measure on $S^{n-1}$. For a Radon measure $\mu$ on $\mathbb{R}^{n}$ the pushforward $\Phi_{*}(\mu \times \omega)$ is a measure on $\mathcal{H}$.

Example 4.1. Let $\mu$ be a nonatomic measure (meaning $\mu(\{x\})=0$ for every $x$ ) such that the support of $\mu$ is not contained in any line. If

$$
0<\int_{\mathbb{R}^{n}}|x|^{-1} d \mu(x)<\infty,
$$

then $\Phi_{*}(\mu \times \omega)$ satisfies the assumptions of Definition 1.1. If, in addition, $\mu$ is a doubling measure, then $\Phi_{*}(\mu \times \omega)$ is uniformly transverse.

Recall that a measure $\mu$ is doubling if there exists a constant $C$ such that $\mu(B(x, r)) \leq C \mu(B(x, r))$ for all $x \in \mathbb{R}^{n}$ and all $r>0$.

Proof. For every $x \in \mathbb{R}^{n}$ and $r>0$ we have

$$
\nu(\pi B(x, r)) \leq \mu(B(x, r))+C \int_{\mathbb{R}^{n}} \frac{r}{|x-y|} d \mu(y)
$$

which implies that the first and third conditions in Definition 1.1 hold. The second condition, $\nu(\pi[x, y])>0$, follows from the support of $\mu$ not being contained in the line through $x$ and $y$.

Suppose $\mu$ is doubling. Fix distinct points $x$ and $y$ and let $r=|x-y|$. Also, fix a unit vector $w$ that is orthogonal to $x-y$. For $k=1,2, \ldots$ let $\mu_{k}$ be the restriction of $\mu$ to the spherical shell $A_{k}=B\left(x, 2^{k+1} r\right) \backslash B\left(x, 2^{k} r\right)$. This shell contains the open ball $B_{k}=B\left(x+3 \cdot 2^{k-1} w, 2^{k-1}\right)$. The doubling condition implies that $\mu\left(B_{k}\right) \geq c \mu\left(A_{k}\right)$ with $c$ independent of $k$. It is geometrically evident that every hyperplane $H$ that meets both $[x, y]$ and $B_{k}$ satisfies $\alpha(x-y, H) \geq \pi / 4$. Thus, the measure $\nu_{k}=\Phi_{*}\left(\mu_{k} \times \omega\right)$ satisfies

$$
\int_{\pi[x, y]} \sin \alpha(x-y, H) d \nu_{k}(H) \geq c \nu_{k}(\pi[x, y])
$$

with $c$ independent of $k$. Observe also that the restriction of $\mu$ to $B(x, 2 r)$, which is not included in any $\mu_{k}$, is comparable in mass to $\mu_{1}$; thus its contribution to $\nu(\pi[x, y])$ is controlled by (4.2) with $k=1$. Summing over $k$, we conclude that $\nu$ is uniformly transverse. 
When $\nu=\Phi_{*}(\mu \times \omega)$, the formula (2.1) yields

$$
f_{\nu}(x)=c \int_{\mathbb{R}^{n}}\left(\frac{x-y}{|x-y|}+\frac{y-o}{|y-o|}\right) d \mu(y)
$$

with some constant factor $c>0$. Indeed, it suffices to verify (4.3) for a unit point mass $\delta_{a}$ because general measures can be approximated by linear combinations of point masses. In turn, $\delta_{a}$ is the limit of normalized restrictions of the Lebesgue measure to $B(a, r)$ as $r \rightarrow 0$. If the basepoint $o$ in the definition of $f_{\nu}$ coincides with $a$, a symmetry consideration yields

$$
\int_{\pi[0, x]} n(H) d \nu(H)=c \frac{x}{\|x\|}, \quad|x|>r
$$

with $c$ independent of $x$ or $r$. Changing the basepoint $o$ contributes additive constants to $f_{\nu}$ and to the right side of (4.3). Since both sides of (4.3) turn to 0 when $x=o$, the additive constants agree. This proves (4.3).

The integral (4.3) was used in [13] to construct quasiconformal maps from doubling measures. Thus, Example 4.1 shows that the results of $₫ 3$ recover some of the main results of 13 .

Beurling and Ahlfors [5] proved that every quasisymmetric self-map of $\mathbb{R}$ extends to a quasisymmetric self-map of $\mathbb{R}^{2}$. Up to orientation, quasisymmetric maps on a line are precisely indefinite integrals of doubling measures of $\mathbb{R}$. The following proposition shows that the Beurling-Ahlfors extension can be obtained from Theorem 3.7

Example 4.2. Let $\mu$ be a doubling measure on the real axis $\mathbb{R}$ of the complex plane $\mathbb{C} \approx \mathbb{R}^{2}$. Denote by $\widetilde{\omega}$ the restriction of the arclength measure on $S^{1}$ to the set of unit vectors $\left(v_{1}, v_{2}\right)$ such that $v_{2} \geq \frac{\sqrt{3}}{2}$. Let $\nu=\Phi_{*}(\mu \times \widetilde{\omega})$ with $\Phi$ as above. Then $\nu$ is uniformly transverse.

Moreover, $f_{\nu}: \mathbb{R}^{2} \rightarrow \mathbb{R}^{2}$ is a quasiconformal map such that $f_{\nu}(\mathbb{R})=\mathbb{R}$ and for all $s, t \in \mathbb{R}, s<t$, we have $f_{\nu}(t)-f_{\nu}(s)=\mu([s, t])$.

Observe that every line $H$ in the support of $\nu$ crosses $\mathbb{R}$ at an angle of at least $\pi / 3$.

Proof. Fix two distinct points $x$ and $y$. If the angle that $x-y$ forms with the real axis is less than $\pi / 4$, the uniform transversality condition holds for the segment $[x, y]$ by the construction of $\nu$. Suppose that this angle is at least $\pi / 4$. By partitioning the segment $[x, y]$, we may assume that $\operatorname{dist}([x, y], \mathbb{R}) \geq|x-y|$. Also without loss of generality, $\operatorname{dist}(y, \mathbb{R})>\operatorname{dist}(x, \mathbb{R})$.

Let $I$ be the segment on $\mathbb{R}$ formed by the intersection points of $\mathbb{R}$ with the lines that meet $[x, y]$ at an angle less than $\pi / 12$. Note that $I$ is the base of a triangle with vertex $y$ in which the angle at $y$ is $\pi / 6$ and the segment $[x, y]$ bisects this angle. Let $p$ be the nearest endpoint of $I$ to $y$; if the endpoints are equidistant from $y$ (i.e., $[x, y]$ is vertical), pick either one. Let $I^{\prime} \subset \mathbb{R}$ be the segment of the same length as $I$ and such that $I \cap I^{\prime}=\{p\}$. The doubling condition implies $\mu\left(I^{\prime}\right) \geq c \mu(I)$. It follows that the restriction of $\mu$ to $I^{\prime}$ is responsible for a certain fraction of $\nu(\pi[x, y])$; and since the lines that intersect both $I^{\prime}$ and $[x, y]$ form the angle of at least $\pi / 12$ with the latter, the measure $\nu$ is uniformly transverse.

The quasiconformality of $f_{\nu}$ follows from Theorem 3.7. The fact that $f_{\nu}(\mathbb{R})=\mathbb{R}$ is a consequence of the symmetry of $\nu$ : reflection of the plane across the real axis 
leaves $\nu$ invariant. Finally, for real $s<t$ the definition of $f_{\nu}$ yields

$$
f_{\nu}(t)-f_{\nu}(s)=\int_{s}^{t} \int_{-\pi / 6}^{\pi / 6} \cos \theta d \theta d \mu=\mu([s, t]) .
$$

\section{CONCluding REMARKS}

Theorem 1.2 leads to several natural questions. The main result of Pogorelov's book [15] is that sufficiently smooth projective metrics on $\mathbb{R}^{3}$ can be obtained as $d_{\nu}$ with $\nu$ being a signed measure on $\mathcal{H}$. Szabó [19] extended this result to all dimensions. Although the definition of our map $f_{\nu}$ makes sense when $\nu$ is a signed measure, all results of this paper rely on $\nu$ being positive.

Question 5.1. Can Theorem 1.2 be extended to signed measures $\nu$ that generate positive metrics $d_{\nu}$ ?

A well-known necessary condition for a metric space $X$ to have a bi-Lipschitz embedding into a Euclidean space is that $X$ is doubling, but this condition is not sufficient in general [9]. A projective metric need not be doubling. For example, the Beltrami-Klein model of the hyperbolic space is a nondoubling projective metric on the unit ball of $\mathbb{R}^{n}$, since the hyperbolic space fails the doubling condition. More generally, Hilbert geometries on convex domains are typically Gromov hyperbolic [4, 11 .

Question 5.2. Does every doubling projective metric on a convex domain $\Omega \subseteq \mathbb{R}^{n}$ admit a bi-Lipschitz embedding into some $\mathbb{R}^{N}$ ? Or even into $\mathbb{R}^{n}$ ?

\section{ACKNOWLEDGEMENT}

The authors thanks the referee for valuable comments and corrections.

\section{REFERENCES}

[1] Ralph Alexander, Planes for which the lines are the shortest paths between points, Illinois J. Math. 22 (1978), no. 2, 177-190. MR490820(82d:53042)

[2] Juan C. Álvarez Paiva, Hilbert's fourth problem in two dimensions, MASS selecta, Amer. Math. Soc., Providence, RI, 2003, pp. 165-183. MR2027175 (2004k:52002)

[3] R. V. Ambartzumian, Combinatorial solution of the Buffon-Sylvester problem, $\mathrm{Z}$. Wahrscheinlichkeitstheorie und Verw. Gebiete 29 (1974), 25-31. MR0372939 (51 \#9143)

[4] Yves Benoist, Convexes hyperboliques et fonctions quasisymétriques (French, with English summary), Publ. Math. Inst. Hautes Études Sci. 97 (2003), 181-237, DOI 10.1007/s10240003-0012-4. MR2010741 (2005g:53066)

[5] A. Beurling and L. Ahlfors, The boundary correspondence under quasiconformal mappings, Acta Math. 96 (1956), 125-142. MR0086869(19,258c)

[6] Herbert Busemann, Geometries in which the planes minimize area, Ann. Mat. Pura Appl. (4) 55 (1961), 171-189. MR0143155 (26 \#715)

[7] Herbert Busemann, Problem IV: Desarguesian spaces, Mathematical developments arising from Hilbert problems (Proc. Sympos. Pure Math., Northern Illinois Univ., De Kalb, Ill., 1974), Amer. Math. Soc., Providence, R. I., 1976, pp. 131-141. Proc. Sympos. Pure Math., Vol. XXVIII. MR0430935 (55 \#3940)

[8] Herbert Busemann and Paul J. Kelly, Projective geometry and projective metrics, Academic Press Inc., New York, N. Y., 1953. MR0054980 (14,1008e)

[9] Juha Heinonen, Lectures on analysis on metric spaces, Universitext, Springer-Verlag, New York, 2001. MR:1800917 (2002c:30028)

[10] Tomi J. Laakso, Plane with $A_{\infty}$-weighted metric not bi-Lipschitz embeddable to $\mathbb{R}^{N}$, Bull. London Math. Soc. 34 (2002), no. 6, 667-676, DOI 10.1112/S0024609302001200. MR1924353 (2003h:30029) 
[11] Anders Karlsson and Guennadi A. Noskov, The Hilbert metric and Gromov hyperbolicity, Enseign. Math. (2) 48 (2002), no. 1-2, 73-89. MR1923418(2003f:53061)

[12] Leonid V. Kovalev, Quasiconformal geometry of monotone mappings, J. Lond. Math. Soc. (2) 75 (2007), no. 2, 391-408, DOI 10.1112/jlms/jdm008. MR2340234(2008e:47121)

[13] Leonid Kovalev, Diego Maldonado, and Jang-Mei Wu, Doubling measures, monotonicity, and quasiconformality, Math. Z. 257 (2007), no. 3, 525-545, DOI 10.1007/s00209-007-01325. MR2328811 (2008e:30030)

[14] Athanase Papadopoulos, On Hilbert's fourth problem, available at arXiv:1312.3172.

[15] Aleksei Vasil'evich Pogorelov, Hilbert's fourth problem, V. H. Winston \& Sons, Washington, D.C.; A Halsted Press Book, John Wiley \& Sons, New York-Toronto, Ont.-London, 1979. Translated by Richard A. Silverman; Scripta Series in Mathematics. MR 550440 (80j:53066)

[16] R. Tyrrell Rockafellar, Convex analysis, Princeton Landmarks in Mathematics, Princeton University Press, Princeton, NJ, 1997. Reprint of the 1970 original; Princeton Paperbacks. MR $1451876(97 \mathrm{~m}: 49001)$

[17] Rolf Schneider and Wolfgang Weil, Stochastic and integral geometry, Probability and its Applications (New York), Springer-Verlag, Berlin, 2008. MR2455326 (2010g:60002)

[18] Stephen Semmes, On the nonexistence of bi-Lipschitz parameterizations and geometric problems about $A_{\infty}$-weights, Rev. Mat. Iberoamericana 12 (1996), no. 2, 337-410, DOI 10.4171/RMI/201. MR1402671 (97e:30040)

[19] Z. I. Szabó, Hilbert's fourth problem. I, Adv. in Math. 59 (1986), no. 3, 185-301, DOI 10.1016/0001-8708(86)90056-3. MR835025 (88f:53113)

[20] Jussi Väisälä, Lectures on n-dimensional quasiconformal mappings, Lecture Notes in Mathematics, Vol. 229, Springer-Verlag, Berlin-New York, 1971. MR0454009 (56 \#12260)

215 Carnegie, Mathematics Department, Syracuse University, Syracuse, New York 13244

E-mail address: 1vkovale@syr.edu 